\title{
Comparison among mid-row soil managements techniques to preserve grape quality in Cabernet Sauvignon vines
}

\author{
Francesca Paoli ${ }^{1, *}$, Linda Salvi ${ }^{1}$, Eleonora Cataldo ${ }^{1}$, Sofia Sbraci $^{1}$, Rita Perria $^{2}$, Paolo Storchi ${ }^{2}$, and \\ Giovan Battista Mattii ${ }^{1}$ \\ ${ }^{1}$ DISPAA, Department of Agri-Food and Environmental Science, University of Florence - Sesto Fiorentino, 50019, Italy \\ ${ }^{2}$ CREA, Research Centre for Forestry and Wood - Arezzo, 52100, Italy
}

\begin{abstract}
Conservative technique of soil management plays an important role, compared to conventional tillage, in order to save soil fertility, to preserve biodiversity and to maintain vegetative-productive balance. However, alongside the many agronomic and environmental benefits, it is worth to consider that grass cover exerts water and nutritional competition, especially where irrigation is difficult to set up. This research is aimed to evaluate the effects, on vines physiology and quality of the grapes, of three different soil management: temporary sward over all interrows (IT), green manure and tillage every other row (SL) and mulching with plant residues and tillage every other row (PL); measurements were carried out in 2016 and 2017 on a Cabernet Sauvignon vineyard, in Bolgheri (Tuscany). During the experiment, single leaf gas exchanges, stem water potential, productivity were measured, while quality parameters were analyzed. Significant differences in gas exchanges related to the different season and inter-row management were observed. Compared to SL and PL, the IT showed more negative values of water potential, due to the grass-vine competition, especially when water availability is greater. The competition, exerted by sward, at the beginning of the season 2017, lead to differences in fruit setting with impact on yield; therefrom, significant differences also in anthocyanic content.
\end{abstract}

\section{Introduzione}

La gestione del suolo vitato riveste un ruolo di fondamentale importanza nel contenimento della flora spontanea, la quale esercita una competizione dal punto di vista idrico e nutrizionale con la pianta [1].

Le lavorazioni convenzionali, pur consentendo un controllo del regime idrico del suolo, limitando le perdite di acqua per evaporazione, favoriscono l'erosione, specialmente nei terreni declivi, e tendono a danneggiare le radici superficiali della vite, con gravi ripercussioni sull'esplorazione dello strato di terreno più fertile e ricco di sostanza organica. Inoltre, peggiorano la struttura e facilitano la formazione della suola di lavorazione [1]. La moderna viticoltura si prefigge un approccio maggiormente conservativo, al fine di tutelare l'equilibrio vegeto-produttivo della pianta, la sostenibilità ambientale ed economica del vigneto, la biodiversità, la composizione del suolo e la qualità delle uve [2]. A tal proposito, la pratica dell'inerbimento (permanente $o$ temporaneo, totale o parziale, spontaneo o seminato) offre numerosi vantaggi: incrementa, nel medio-lungo termine, la sostanza organica del suolo, favorendo una maggiore disponibilità di nutrienti per la pianta, per mezzo di un miglioramento della capacità di scambio cationico [3]; previene i processi erosivi e la lisciviazione dei nitrati nelle falde [4]; migliora lo stato di aggregazione delle particelle del suolo, quindi la permeabilità e la portanza del terreno, consentendo l'ingresso tempestivo dei mezzi in campo; ottimizza la disponibilità annua dell'azoto. Oltre a ciò, risulta economicamente più conveniente, sia dal punto di vista dei consumi energetici, sia della richiesta di manodopera [5].

Bisogna poi considerare che, se da un lato la competizione idrica e nutrizionale tra pianta e tappeto erboso può ridurre fino al $30 \%$ la vigoria e la produzione nel vigneto $[1 ; 6]$, dall'altro può causare squilibri nelle fasi di sviluppo delle piante, specialmente nei primi anni di impostazione dell'inerbimento [5].

L'obiettivo di questa ricerca, svolta in due annate consecutive, è stato verificare gli effetti di tre diverse gestioni dell'interfilare sulla fisiologia della pianta e sulla qualità delle uve.

\section{Materiali e metodi}

La ricerca si è svolta nell'Alta Maremma toscana, nella DOC di Bolgheri (Livorno). L'area, mitigata dall'azione dei venti e dalla vicinanza del mare, che contribuisce, per riflesso, a garantire anche una buona illuminazione, è caratterizzata da un clima mediterraneo, con piovosità media annua di circa $600 \mathrm{~mm}$ e temperatura media annua intorno ai $14{ }^{\circ} \mathrm{C}$. Il terreno ha una prevalenza di argille 
plioceniche sottili. Il vigneto oggetto di sperimentazione si sviluppa su una superficie di 3,45 ettari, coltivati a Cabernet Sauvignon; è stato impiantato nel 2007, con sesto d'impianto 2,00 x 0,70 m ed esposizione NordNord Est/Sud-Sud Ovest; le viti, innestate su 420A, sono allevate a controspalliera e potate a cordone speronato. La ricerca è stata condotta nelle stagioni 2016 e 2017 , impostando tre diverse gestioni dell'interfilare in un disegno a blocchi completamente randomizzati, costituito da 5 blocchi di 15 interfilari ciascuno. Le tre tesi (5 interfilari/tesi per blocco), corrispondenti alle diverse gestioni dell'interfilare, sono le seguenti:

- IT, inerbimento spontaneo su tutti gli interfilari;

- SL, sovescio classico e lavorazione superficiale a $20 \mathrm{~cm}$ di profondità, a interfilari alterni. Il sovescio è realizzato con trinciatura e interramento annuale di essenze spontanee;

- PL, pacciamatura e lavorazione superficiale, a interfilari alterni. La pacciamatura consiste nella creazione di uno strato pacciamante naturale, con residui vegetali spontanei, ottenuto tramite schiacciamento della biomassa.

Nel 2016, sono state effettuate due trinciature della flora spontanea: a fine maggio e a fine giugno, mentre nel 2017 solo a inizio giugno, all'epoca della fioritura. Nelle stesse epoche, sono state svolte le lavorazioni negli interfilari e lo schiacciamento della vegetazione spontanea.

Allo scopo di analizzare gli scambi gassosi, nelle principali fasi fenologiche e a seguito di eventi climatici rilevanti, dalla fine di Maggio fino alla vendemmia, sono stati effettuati rilievi di fotosintesi (Pn) e conduttanza stomatica (gs) su foglia singola, per mezzo di analizzatore differenziale di gas ad infrarossi, CIRAS-3 (PP Systems, USA); a mezzogiorno, sulle stesse foglie utilizzate per i rilievi di scambi gassosi, è stato misurato il potenziale idrico di tralcio $\left(\Psi_{\mathrm{ms}}\right.$ : midday stem water potential), tramite camera a pressione di Scholander (600 EXP, PMS Instrument Company). A partire dall'invaiatura, con cadenza settimanale, sono stati prelevati campioni di $100 \mathrm{acini} /$ ripetizione, per le analisi qualitative tecnologiche: peso medio dell'acino (g); grado zuccherino ( ${ }^{\circ}$ Brix), determinato con rifrattometro ottico manuale (N-8 alpha, ATAGO); acidità totale $(\mathrm{g} / \mathrm{L}$ ac. tartarico), ottenuta per titolazione a $\mathrm{pH} 7$ con $\mathrm{NaOH}$ $\mathrm{N} / 10$, utilizzando come indicatore il blu di bromotimolo; $\mathrm{pH}$, rilevato con metodo potenziometrico ed analisi fenoliche: contenuto antocianico e polifenolico, totale ed estraibile $(\mathrm{mg} / \mathrm{L})$, tramite metodo Glories. Alla vendemmia, su 10 piante per tesi/ripetizione, sono stati rilevati i dati relativi alla produzione: numero di acini a grappolo, numero di grappoli a pianta, peso medio del grappolo $(\mathrm{g})$, resa per pianta $(\mathrm{kg})$. I dati sono stati poi sottoposti ad analisi della varianza ad una via ed il test Least Significant Difference (LSD) di Fisher è stato calcolato, con un livello di significatività di 0,05 , tramite il software SPSS Statistics.

\section{Risultati e discussioni}

Le due annate di sperimentazione sono state caratterizzate da andamenti climatici molto diversi tra loro. Il 2016 (Fig. 1) ha visto precipitazioni sopra la media nei mesi di maggio e giugno, che si sono protratte, seppur in misura più contenuta, durante il periodo più caldo della stagione, con temperature massime in linea con la media stagionale, consentendo il raggiungimento di un'ottimale livello di maturità delle uve alla vendemmia (4 ottobre 2016).

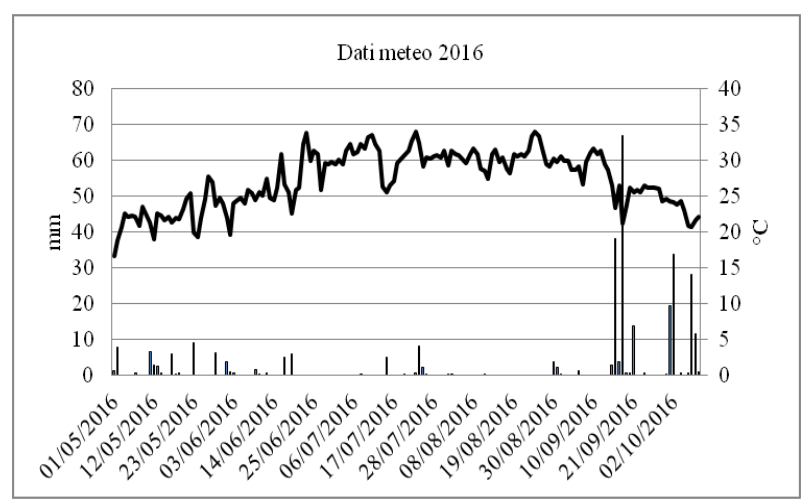

Fig. 1. Andamento meteorologico stagione 2016. Il grafico mette in evidenza l'andamento delle temperature massime $\left({ }^{\circ} \mathrm{C}\right)$ e delle precipitazioni giornaliere $(\mathrm{mm})$.

Il 2017 (Fig. 2), invece, è stato caratterizzato da temperature minime molto basse a fine Aprile $\left(2,1^{\circ} \mathrm{C}\right)$, cui sono seguite temperature massime molto elevate (con un picco massimo di $38,7{ }^{\circ} \mathrm{C}$ ad inizio agosto) ed assenza pressoché totale di eventi piovosi da maggio fino ai primi di settembre, momento in cui, visto lo stato delle uve e considerate le previsioni meteorologiche, si è preferito procedere alla raccolta (8 settembre 2017).

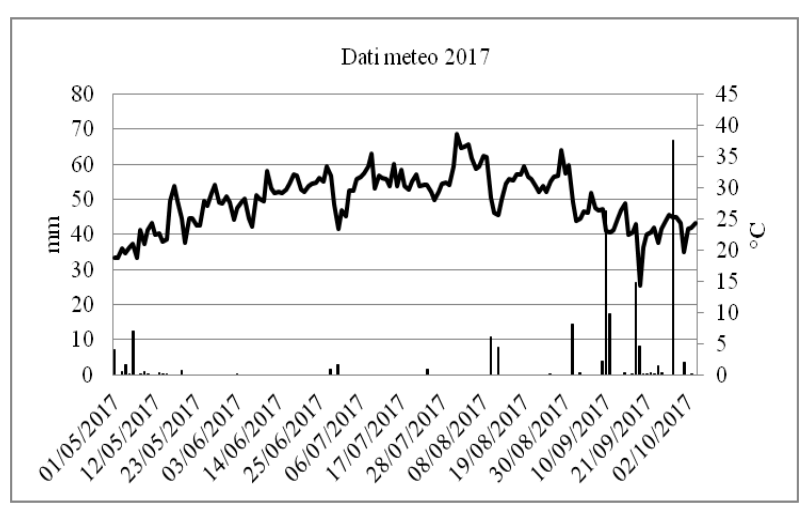

Fig. 2. Andamento meteorologico stagione 2017. Il grafico mette in evidenza l'andamento delle temperature massime $\left({ }^{\circ} \mathrm{C}\right)$ e delle precipitazioni giornaliere $(\mathrm{mm})$.

Nelle Figg. 3, 4, 5, 6, si osservano gli andamenti di fotosintesi netta e conduttanza stomatica, relativi ai due anni di rilievo. Nel 2016, la tesi IT ha mostrato valori di fotosintesi netta tendenzialmente più bassi rispetto alle altre tesi (Fig. 3), soprattutto in prossimità di periodi piovosi; inoltre, sono stati evidenziati valori di conduttanza stomatica significativamente inferiori nella tesi IT rispetto a SL e PL (fine giugno-inizio luglio e 8 settembre) (Fig. 4). 


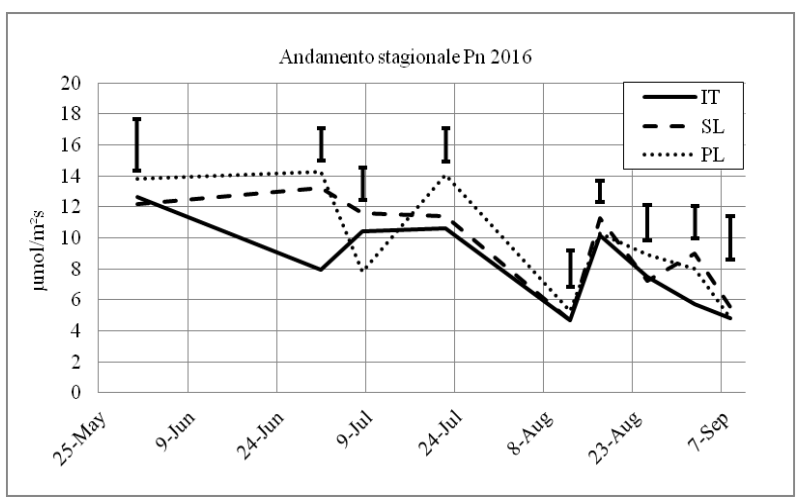

Fig. 3. Andamento stagionale della fotosintesi netta $(\mathrm{Pn})$ nel 2016. Le barre indicano la differenza minima significativa (LSD), ottenuta tramite ANOVA a una via $(\mathrm{P} \leq 0.05)$.

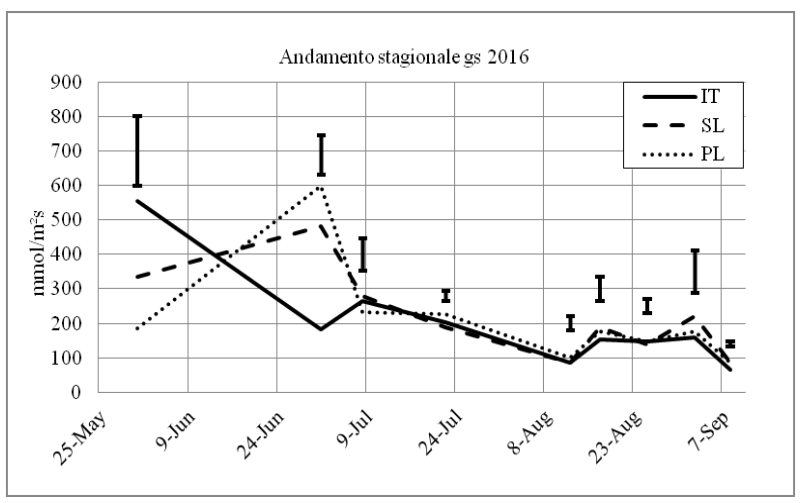

Fig. 4. Andamento stagionale della conduttanza stomatica (gs) nel 2016. Le barre indicano la differenza minima significativa (LSD), ottenuta tramite ANOVA a una via $(\mathrm{P} \leq 0.05)$.

Nel 2017, a causa della stagione calda e siccitosa, si riscontrano differenze meno marcate tra le tesi ed i valori tendono ad allinearsi maggiormente per entrambi i parametri; tuttavia, nei rilievi della prima metà di luglio 2017, la tesi IT ha evidenziato valori generalmente minori di fotosintesi netta rispetto a SL e PL, che, invece, si attestano su valori simili tra loro (Fig. 5). Per quanto riguarda la conduttanza stomatica, nella parte finale della stagione, tutte le tesi hanno mostrato lo stesso trend, collocandosi in un range di valori indicativi di uno stress moderato [7] (Fig. 6).

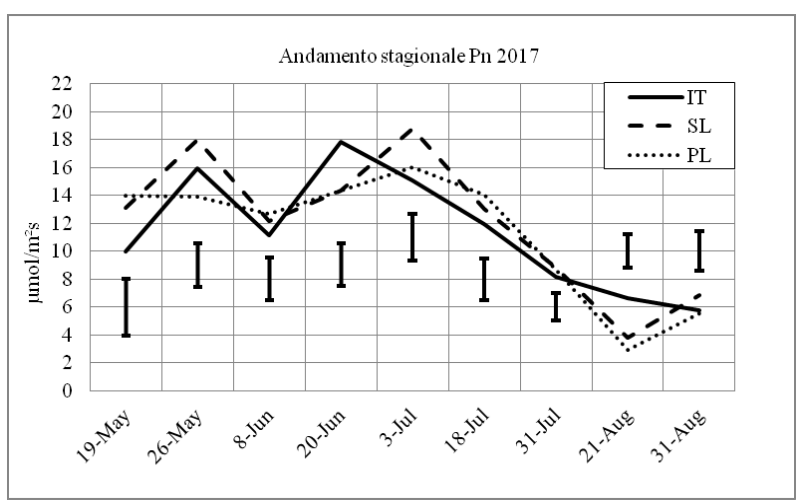

Fig. 5. Andamento stagionale della fotosintesi netta (Pn) nel 2017. Le barre indicano la differenza minima significativa (LSD), ottenuta tramite ANOVA a una via $(\mathrm{P} \leq 0.05)$.

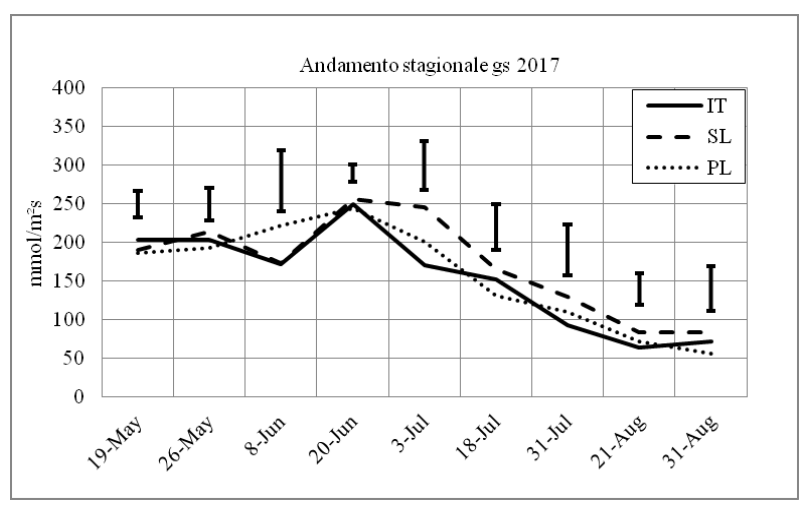

Fig. 6. Andamento stagionale della conduttanza stomatica (gs) nel 2017. Le barre indicano la differenza minima significativa (LSD), ottenuta tramite ANOVA a una via $(\mathrm{P} \leq 0.05)$.

Nella valutazione del potenziale idrico di tralcio a mezzogiorno $\left(\Psi_{\mathrm{ms}}\right)$, in linea generale, si possono osservare valori che indicano un miglior status idrico delle piante nel 2016, rispetto al 2017. Come si osserva in Fig. 7, nella prima annata di rilievi, la tesi IT ha evidenziato uno stress maggiore, nei periodi caratterizzati da una moderata piovosità (22-29 luglio e 2 settembre 2016); lo stesso andamento è stato confermato anche nel 2017 (ultima settimana di maggio-prima di giugno e dalla seconda settimana di agosto in poi) (Fig. 8).

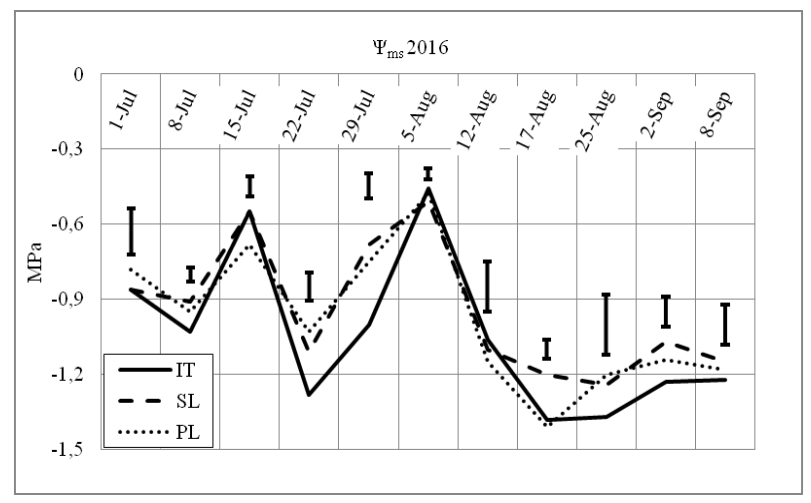

Fig. 7. Andamento stagionale del potenziale idrico di tralcio $\left(\Psi_{\mathrm{ms}}\right)$, misurato al mezzogiorno, nel 2016. Le barre indicano la differenza minima significativa (LSD), ottenuta tramite ANOVA a una via $(\mathrm{P} \leq 0.05)$. 


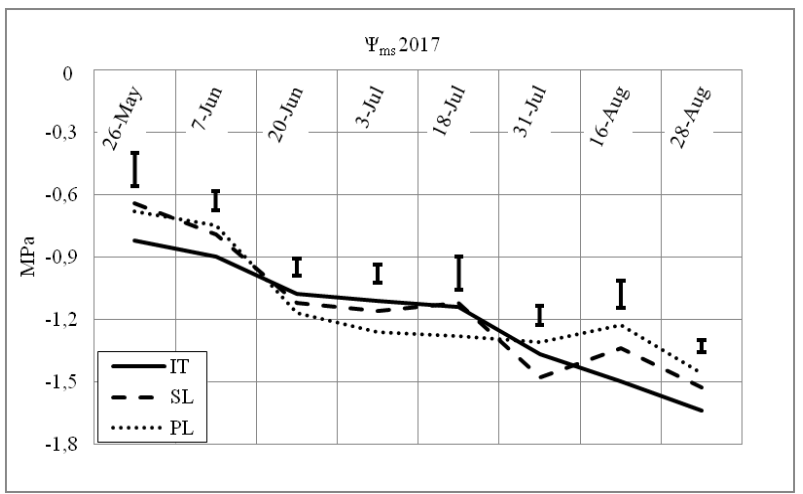

Fig. 8. Andamento stagionale del potenziale idrico di tralcio $\left(\Psi_{\mathrm{ms}}\right)$, misurato al mezzogiorno, nel 2017. Le barre indicano la differenza minima significativa (LSD), ottenuta tramite ANOVA a una via $(\mathrm{P} \leq 0.05)$.

Complessivamente, dai dati degli scambi gassosi e del potenziale idrico nelle due annate, è emerso uno stress più elevato nella tesi IT, rispetto alle altre tesi; al contrario, i valori delle tesi SL e PL tendono spesso a sovrapporsi. Questo trend si evidenzia specialmente nei periodi in prossimità di eventi piovosi, mentre, nella parte centrale della stagione, più calda e secca, l'andamento è risultato più costante, pur rivelando differenze. Si suppone, quindi, che la maggiore disponibilità idrica, seppur moderata, abbia favorito la ripresa vegetativa del cotico erboso, favorendo una maggior competizione idrica tra la pianta ed il tappeto erboso.

Per quanto riguarda i parametri produttivi (Tab. 1), nel 2016, il peso medio dell'acino è risultato significativamente inferiore nella tesi IT $(1,01 \mathrm{~g})$ rispetto alle tesi SL (1,25 g) e PL (1,29 g); al contrario, nel 2017, è stata la tesi PL $(0,61 \mathrm{~g})$ a mostrare valori più bassi di IT $(0,77 \mathrm{~g})$. Altre differenze sono state riscontrate nel numero di acini a grappolo che, nel 2016, è risultato significativamente superiore per la tesi IT $(113,26)$ in confronto alle tesi SL $(93,84)$ e PL $(84,91)$; nel 2017, invece, la tesi SL $(84,22)$ ha evidenziato valori superiori rispetto a IT $(60,29)$. Per quanto riguarda il peso medio del grappolo, non sono presenti differenze significative nel primo anno di rilievi, mentre nel 2017 la tesi SL $(65,86 \mathrm{~g})$ si è rivelata superiore rispetto a IT $(48,87 \mathrm{~g}) \mathrm{e}$ PL (47,51 g). Nessuna differenza significativa è stata riscontrata nel numero di grappoli a pianta, in nessuna delle due annate (dati non presentati). La resa per pianta non ha presentato differenze significative nel 2016, mentre nel 2017, la tesi IT ha mostrato un valore più basso $(0,23 \mathrm{~kg})$ di SL $(0,33 \mathrm{~kg})$.
Tab. 1. Tabella riassuntiva dei parametri produttivi a vendemmia. La differenza minima significativa (LSD), all'interno della stessa annata, è stata ottenuta mediante ANOVA a una via $(\mathrm{P} \leq 0.05)$.

\begin{tabular}{|c|c|c|c|c|c|}
\cline { 2 - 6 } \multicolumn{1}{c|}{2016} & Tesi & $\begin{array}{c}\text { Peso medio } \\
\text { dell'acino } \\
(\mathrm{g})\end{array}$ & $\begin{array}{c}\mathrm{N}^{\circ} \text { acini / } \\
\text { grappolo }\end{array}$ & $\begin{array}{c}\text { Peso medio } \\
\text { del } \\
\text { grappolo } \\
(\mathrm{g})\end{array}$ & $\begin{array}{c}\text { Resa / } \\
\text { pianta } \\
(\mathrm{kg})\end{array}$ \\
\hline & $\mathrm{IT}$ & 1,01 & 113,26 & 120,79 & 0,9 \\
\cline { 2 - 6 } & $\mathrm{SL}$ & 1,25 & 93,84 & 123,75 & 1 \\
\cline { 2 - 6 } & PL & 1,29 & 84,91 & 115 & 0,9 \\
\cline { 2 - 6 } & LSD & 0,13 & 17,94 & $\mathrm{~ns}$ & $\mathrm{~ns}$ \\
\hline \multirow{4}{*}{2017} & $\mathrm{IT}$ & 0,77 & 60,29 & 48,87 & 0,23 \\
\cline { 2 - 6 } & $\mathrm{SL}$ & 0,74 & 84,22 & 65,86 & 0,33 \\
\cline { 2 - 6 } & PL & 0,61 & 73,54 & 47,51 & 0,27 \\
\cline { 2 - 6 } & LSD & 0,16 & 16,87 & 12,32 & 0,09 \\
\hline
\end{tabular}

Per ciò che riguarda le caratteristiche produttive, conseguentemente alle differenze climatiche nelle due stagioni, nel 2017, in tutte le tesi, si è osservata una sensibile diminuzione nel numero di acini a grappolo e del peso medio dell'acino. A conferma di ciò, i dati della produzione (in ettolitri di vino) rivelano che, nel bolgherese, si è assistito, ad un calo del $25 \%$ circa, rispetto al 2016 [8].

La tesi IT ha evidenziato una maggior suscettibilità alla stagione calda e siccitosa, mostrando nel 2017 un dimezzamento del numero di acini per grappolo rispetto al 2016. Si ipotizza, quindi, che la competizione per risorse idriche, instaurata dal cotico erboso ad inizio stagione 2017, possa aver avuto effetti sull'allegagione, con conseguente riduzione del numero di acini/grappolo rispetto al 2016 e aumento delle dimensioni dell'acino rispetto alle altre tesi. Un'altra causa di ridotta allegagione potrebbe essere ricondotta alle basse temperature di fine aprile, molto prossime a $0{ }^{\circ} \mathrm{C}\left(\mathrm{T}^{\circ}\right.$ $\min =2,1^{\circ} \mathrm{C}$ il $19-20$ aprile 2017 ).

Differenze statisticamente significative sono state riscontrate nella composizione del mosto, solo nel 2016: la tesi IT ha mostrato un maggior contenuto zuccherino ed un $\mathrm{pH}$ più elevato $\left(27,7^{\circ} \mathrm{Brix} \mathrm{e} \mathrm{pH}=3,66\right)$ rispetto a SL $\left(25,6^{\circ}\right.$ Brix e $\left.\mathrm{pH}=3,49\right)$ e $\mathrm{PL}\left(25,6^{\circ}\right.$ Brix e $\mathrm{pH}=$ $3,46)$; l'acidità totale è risultata maggiore nella tesi PL $(4,52 \mathrm{~g} / 1 \mathrm{di}$ ac. tartarico) rispetto a IT e SL (rispettivamente 3,62 e 3,76 g/l di ac. tartarico).

Nel 2016, inoltre, nella tesi IT, è stato rilevato il maggior contenuto in antociani totali $(1845,55 \mathrm{mg} / \mathrm{L})$, statisticamente differente solo rispetto a PL (1535,45 $\mathrm{mg} / \mathrm{L})$; nel 2017, SL ha evidenziato valori significativamente superiori $(2044,5 \mathrm{mg} / \mathrm{L})$ rispetto a IT $(1696,33 \mathrm{mg} / \mathrm{L})$ e PL $(1776,83 \mathrm{mg} / \mathrm{L})$. Nessuna differenza è stata osservata relativamente al contenuto polifenolico totale nei due anni (Tab. 2). 
Tab. 2. Tabella riassuntiva dei parametri tecnologici e fenolici, a vendemmia. La differenza minima significativa (LSD), all'interno della stessa annata, è stata ottenuta mediante ANOVA a una via $(\mathrm{P} \leq 0.05)$.

\begin{tabular}{|c|c|c|c|c|c|c|}
\cline { 2 - 7 } \multicolumn{1}{c|}{} & Tesi & $\begin{array}{c}\text { Solidi } \\
\text { solubili } \\
\left({ }^{\circ} \text { Brix }\right)\end{array}$ & $\begin{array}{c}\text { Acidità } \\
\text { totale } \\
(\mathrm{g} / \mathrm{L})\end{array}$ & $\mathrm{pH}$ & $\begin{array}{c}\text { Antociani } \\
\text { totali } \\
(\mathrm{mg} / \mathrm{L})\end{array}$ & $\begin{array}{c}\text { Polifenoli } \\
\text { totali } \\
(\mathrm{mg} / \mathrm{L})\end{array}$ \\
\hline \multirow{5}{*}{2016} & $\mathrm{IT}$ & 27,7 & 3,62 & 3,66 & 1845,55 & 2872,25 \\
\cline { 2 - 7 } & $\mathrm{SL}$ & 25,6 & 3,76 & 3,49 & 1699,60 & 2813,35 \\
\cline { 2 - 7 } & $\mathrm{PL}$ & 25,6 & 4,52 & 3,46 & 1535,45 & 2690,47 \\
\cline { 2 - 7 } & $\mathrm{LSD}$ & 1,1 & 0,76 & 0,06 & 248,82 & $\mathrm{~ns}$ \\
\hline \multirow{5}{*}{2017} & $\mathrm{IT}$ & 28,5 & 4,67 & 3,64 & 1696,33 & 3607,15 \\
\cline { 2 - 7 } & $\mathrm{SL}$ & 27,9 & 4,83 & 3,58 & 2044,50 & 3976,97 \\
\cline { 2 - 7 } & $\mathrm{PL}$ & 29,5 & 5,10 & 3,53 & 1776,83 & 3854,26 \\
\cline { 2 - 7 } & LSD & $\mathrm{ns}$ & $\mathrm{ns}$ & $\mathrm{ns}$ & 258,87 & $\mathrm{~ns}$ \\
\hline
\end{tabular}

Nei parametri tecnologici e fenolici, nel complesso, si sono osservate più differenze nel 2016 rispetto al 2017, in linea con le variazioni monitorate nei rilievi ecofisiologici.

Nel 2016, il contenuto in solidi solubili ed il pH sono risultati, in senso assoluto, i più elevati nella tesi IT; mentre la ricchezza in antociani totali si è rivelata significativamente maggiore rispetto a PL. Considerando il peso dell'acino osservato nella tesi IT, si ipotizza un possibile effetto concentrazione, dovuto al minor accrescimento della bacca.

Nel 2017, nessuna differenza significativa è stata rilevata nei parametri tecnologici e fenolici, fatta eccezione per il contenuto antocianico totale, significativamente maggiore nella tesi SL, rispetto alle atre tesi. Ciò, potrebbe esser ricondotto ad un deficit idrico in postinvaiatura [9] (Fig. 8, 31 luglio).

\section{Conclusioni}

La ricerca, condotta su due anni, si è prefissata lo scopo di confrontare tre diverse gestioni dell'interfilare (inerbimento totale, sovescio-lavorazione, pacciamaturalavorazione), valutandone gli effetti sulla fisiologia delle piante e sulla qualità delle uve. Dai risultati degli scambi gassosi, è emerso uno stress più elevato nella tesi completamente inerbita, specialmente in presenza di maggior disponibilità idrica, che potrebbe aver stimolato una competizione del tappeto erboso sulla pianta.

Nella seconda annata di rilievi, probabilmente a causa della competizione idrica di inizio stagione o a seguito delle basse temperature di aprile, si è riscontrato un effetto sulla fase di allegagione, più marcato nella tesi inerbita, con conseguente diminuzione del numero di acini a grappolo ed aumento del peso medio dell'acino. Inoltre, a parità di caratteristiche tecnologiche, ove non si registrano differenze, la tesi inerbita ha lo svantaggio di presentare una resa inferiore rispetto alla tesi gestita con sovescio-lavorazione.

Nel terreno sovesciato-lavorato si è evidenziato un contenuto in antociani totali significativamente maggiore; anche il contenuto polifenolico totale, pur non essendo state rilevate differenze, è risultato tendenzialmente superiore.

Gli autori desiderano ringraziare Tenuta dell'Ornellaia per l'opportunità di cooperazione e supporto.

\section{Riferimenti bibliografici}

1. M. Fregoni, Viticoltura di qualità III ed. (Tecniche nuove, 2013)

2. L. Valenti, P. Donna, I. Ghiglieno, L'Informatore Agrario, 5, 36-40 (2014)

3. M. Centinari, L'inerbimento nell'ecosistema vigneto: confronto di metodologie di misura del consumo idrico del prato e risposta allo sfalcio, Alma Mater Studiorum Università di Bologna. Dottorato di ricerca in Colture arboree ed agrosistemi forestali ornamentali e paesaggistici, XX ciclo (2008)

4. S. S. Saladino, Incremento della fertilità dei suoli come conseguenza di pratiche colturali per la gestione sostenibile dei vigneti in climi semi-aridi, Università degli Studi di Palermo. Dottorato di ricerca in Tecnologie per la sostenibilità ed il risanamento ambientale, XXIII ciclo (2011)

5. F. Piras, P. Mulè, M. G. Mameli, L. Demelas, D. Delpiano, R. Zurru, Italus Hortus, , 2, 25-38 (2017)

6. G. Scalabrelli, G. Ferroni, M. Borselli, R. Bandinelli, S. Mancuso, Notiziario tecnico CRPV, 58 (1999)

7. J. Cifre, J. Bota, J.M. Escalona, H. Medrano, J. Flexas, Agriculture, Ecosystems and Environment, 106 159-170 (2005)

8. http://toscana.confagricoltura.it/ita/notizie/news7/vendemmia-annata-difficile-per-il-vino-toscanocalo-del-30-e-una-perdita-di-100-milioni-di-euro (u.c. 28/05/2018)

9. J. A. Kennedy, M. A. Matthews, A. L. Waterhouse, Am J Enol Vitic, 53, 268-274 (2002) 